\title{
The "Texas Longhorn" Syndrome: A Case of Posterior Interosseous Nerve Palsy With Noteworthy Observations
}

\author{
Hassan Kesserwani ${ }^{1}$ \\ 1. Neurology, Flowers Medical Group, Dothan, USA
}

Corresponding author: Hassan Kesserwani, neuro1815@yahoo.com

\begin{abstract}
The posterior interosseous nerve (PIN) is quite a fascinating nerve. It wraps around the radial neck akin to the fibular nerve wrapping around the fibular neck. It can be compressed by the arcade of Frohse in a fashion similar to median nerve entrapment by the carpal tunnel. Furthermore, it divides into a number of branches, a simulacrum of the cauda equina, as it emerges from the supinator muscle. It is also associated with spaceoccupying pathologies; lipomas, and ganglion cysts being among the most common. It also has other interesting anatomical properties, such as fascicular compartmentalization, explaining PIN paralysis with radial nerve injuries. Finally, PIN lesions can lead to partial paralysis of finger extensors leading to the "Texas longhorn" hand gesture. We seize upon a case of PIN entrapment by fibrous adhesions and the arcade of Frohse, and explore the functional anatomy of the PIN.
\end{abstract}

Categories: Neurology, Anatomy

Keywords: peripheral nerve disorders, radial nerve injury

\section{Introduction}

At the radiocapitellar joint of the elbow, the radial nerve bifurcates into the posterior interosseous nerve (PIN) and the superficial radial nerve (SRN). The PIN passes between both heads of the supinator muscle and wraps around the radial neck, in a manner reminiscent of the fibular nerve wrapping around the fibular neck. In $25 \%$ of cases, the PIN contacts the neck of the radius directly over the aptly named "bare area" of the radial neck. Here the PIN supplies the supinator muscle and the extensor carpi radialis brevis (ECRB). As it emerges from the supinator, the PIN divides into multiple divisions resembling a mini-cauda equina. These branches supply the extensor group of muscles of the forearm; extensor digitorum communis (EDC), extensor digiti quinti (EDQ), extensor carpi ulnaris (ECU), abductor pollicis longus (APL), extensor pollicis longus (EPL), extensor pollicis brevis (EPB), and extensor indicis proprius (EIP) [1]. These muscles extend the digits at the metacarpophalangeal joints, ulnar-extend the wrist and extend and abduct the thumb in the plane of the palm, as the names imply. In summary, the PIN supplies all the extensor muscles of the forearm except the extensor carpi radialis longus (ECRL), the radial extensor deviator of the wrist, and the

Review began 01/06/2021 Review ended 01/10/2021 Published 01/18/2021

\section{(c) Copyright 2021}

Kesserwani. This is an open access article distributed under the terms of the Creative Commons Attribution License CC-BY 4.0., which permits unrestricted use, distribution, and reproduction in any medium, provided the original author and source are credited.
Brachioradialis (BR), the elbow flexor with the forearm in 90-degree pronation.

It immediately follows that the PIN does not supply the BR, ECRL, and of course, the triceps, all of which are supplied by the radial nerve proper. Furthermore, the PIN is a purely motor nerve. Therefore, with PIN injury, radial deviation of the wrist and sensation over the SRN distribution are preserved, using useful clinical pearls.

In $30 \%$ of cases, the PIN pierces the superficial head of the supinator and enters an inverted fibrous arch, shaped like a parabola. This is the arcade of Frohse which attaches like a sling from the tip to the medial edge of the lateral epicondyle. The thickness and size of the opening of this arcade are highly variable. The arcade of Frohse is membranous in $68 \%$ and tendinous in $32 \%$ of cases [2]. This arch may press on the PIN in a manner akin to the compression of the median nerve at the carpal tunnel. These three aforementioned anatomic parallels are a useful aid in reminding the clinician of the neuroanatomy of the PIN.

In their 20-year retrospective series of 18 cases, Quignon et al., stress the pattern of finger extension paresis (finger drop) plus radial deviation of wrist extension (due to sparing of the ECRL). Most of their patients were male manual workers. Most cases had a tumor as an etiology, lipoma being the commonest. This was followed by trauma, iatrogenic or idiopathic with equal frequency. Interestingly, in seven cases, entrapment was caused by a sharp tendon of the ECRB. Treatment was frequently done by neurolysis and the prognosis was generally good. Better outcomes were associated with a shorter duration of paresis [3]. The PIN syndrome is also referred to as the supinator syndrome or the radial tunnel syndrome, with subtle distinctions, which we will outline in the Discussion section.

\section{Case Presentation}

We present the case of a 62-year-old right-handed man who presents with vague aching pain of the right 


\section{Cureus}

upper forearm. The weakness of grip progressed over the course of two weeks and the decline halted. In particular, he noted difficulty elevating the ring and middle fingers of the right hand. He denied any radicular symptoms, but noted visible atrophy of the extensor group of muscles of the right forearm. Numbness and tingling were denied. There was no reported trauma.

His past medical history was unremarkable, with no prescribed medications.

The right arm neurologic examination was significant for atrophy of the extensor group of muscles of the right forearm and finger drop of the right middle and ring fingers, with mild weakness of index finger extension, the so-called "Texas longhorn" gesture (Figure 1).

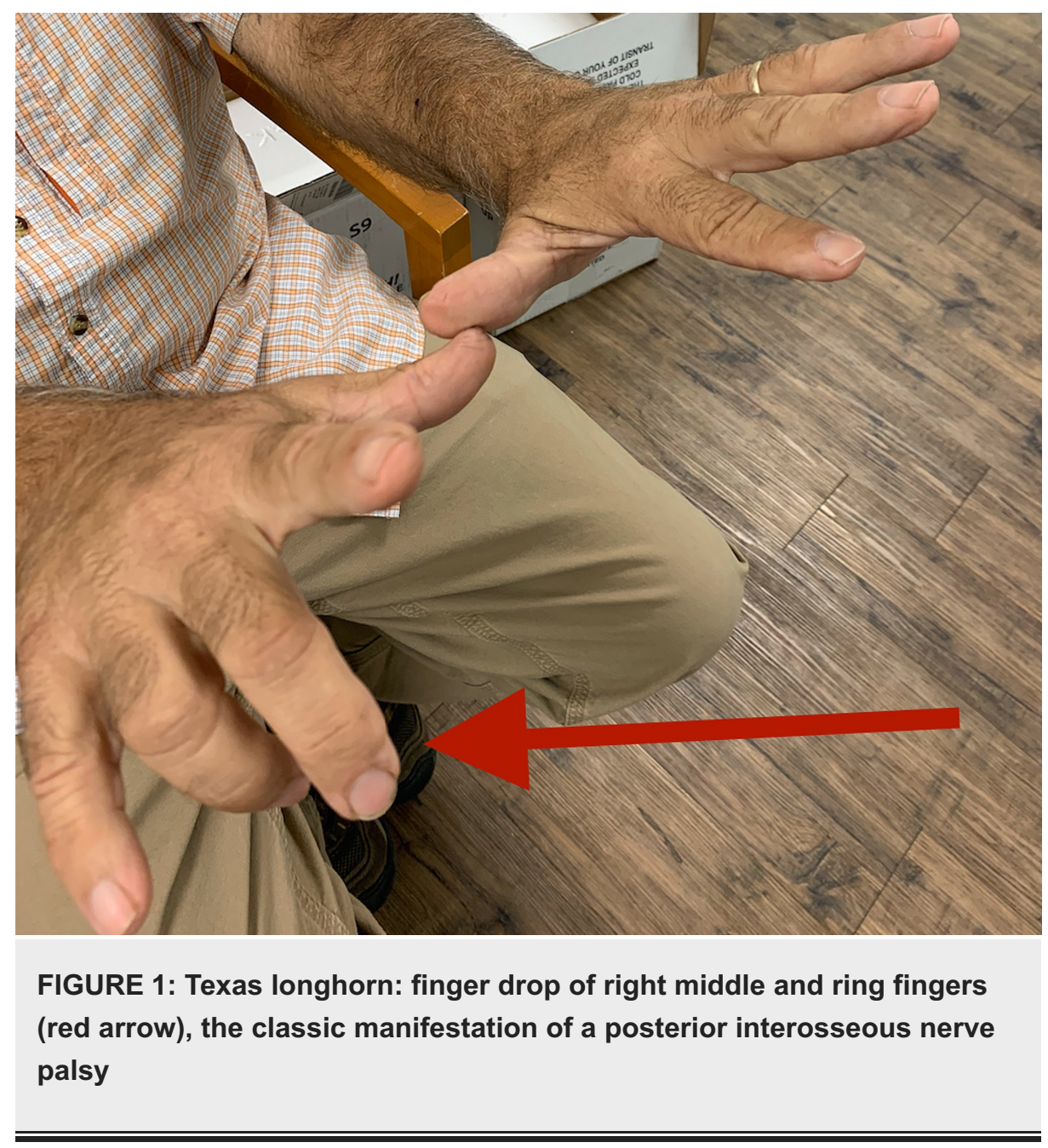

There was also weakness of right thumb extension in the plane of the palm. Right wrist extension and distal interphalangeal extension of all the digits were normal. Deep tendon reflexes including the triceps, BR, and biceps were symmetric in both arms. Sensation over the right superficial radial sensory nerve was preserved to touch and pinprick. Tenderness was elicited by deep compression of the radial (supinator) tunnel over the right supinator muscle. This constellation of findings is highly typical of a right interosseous nerve lesion at the radial tunnel. Particular emphasis should be placed on the preservation of the right ECRL muscle (radially deviated wrist extension), triceps, and BR, all being radial nerve-innervated muscles.

A nerve conduction study (NCS) of the right radial motor nerve, recording from the EIP, revealed a conduction block above the right radial tunnel (Figure 2). 


\section{Cureus}

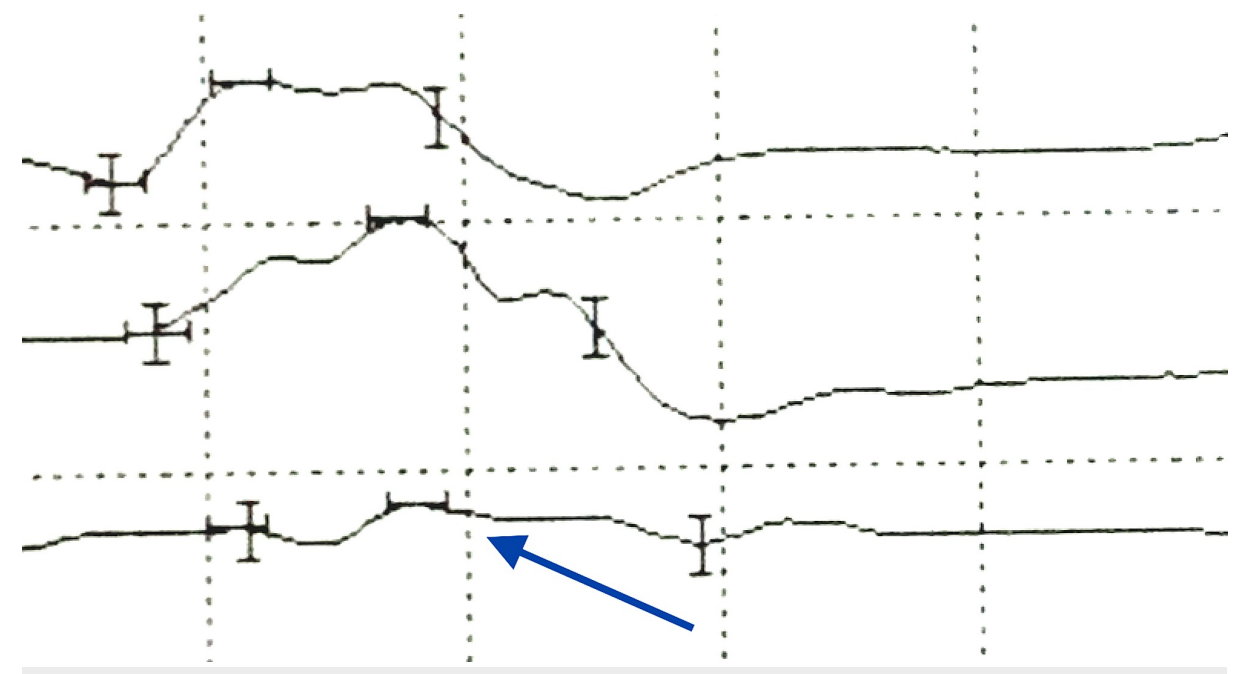

FIGURE 2: Nerve conduction study of posterior interosseous nerve (radial nerve) recording from the extensor indicis proprius; note conduction block with recording above the radial head (blue arrow).

The NCS of the right superficial radial sensory amplitude revealed a normal sensory nerve action potential (SNAP) amplitude, 12 microvolts (normal: greater than or equal to 10 microvolts), with acute denervation of the right EDC on electromyography (EMG) (Figure 3).

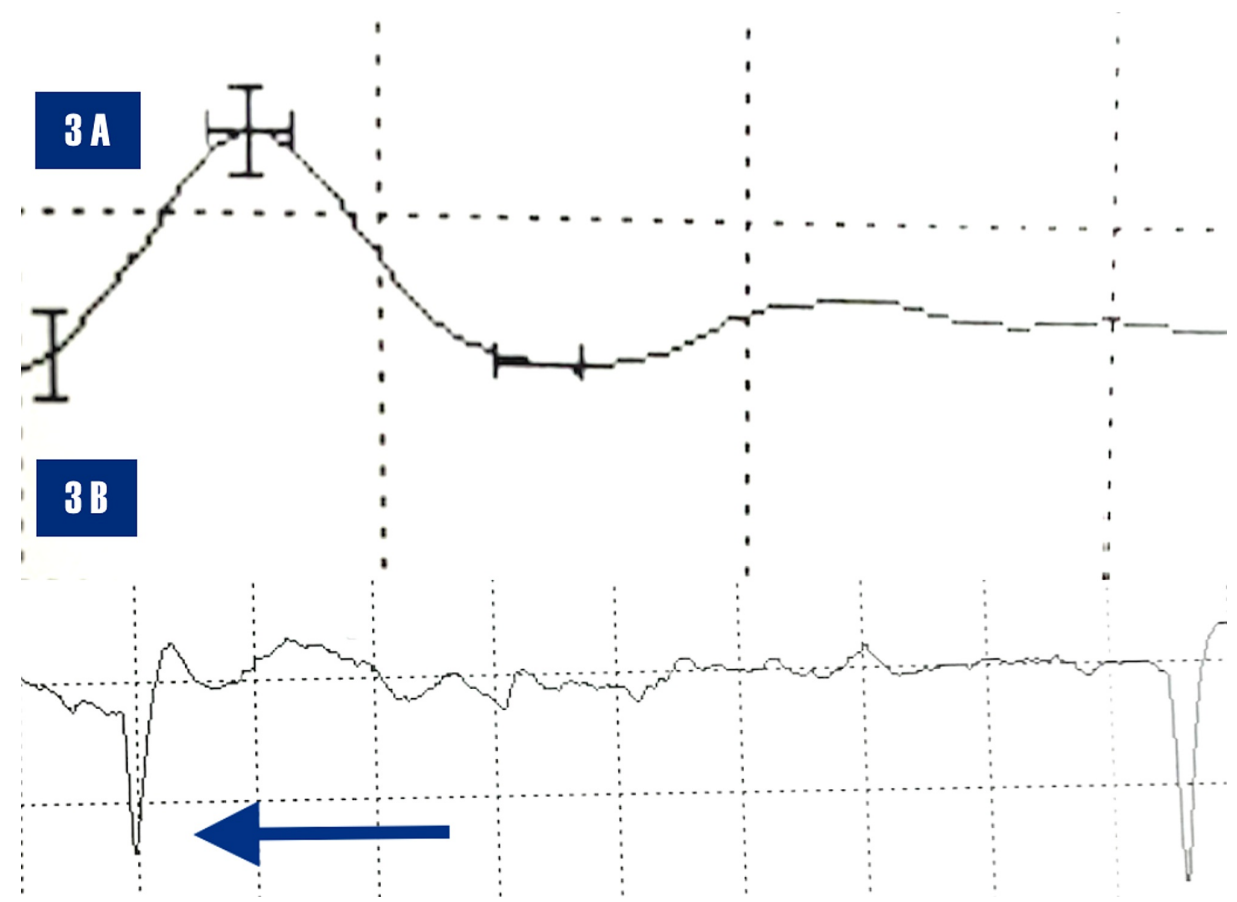

FIGURE 3: 3A. The nerve conduction study demonstrates a normal right radial sensory amplitude of 12 microvolts (normal > 10 microvolts). 3B. Electromyogram of right extensor digitorum communis demonstrates acute denervation, positive waves (blue arrow)

Surgical exploration of the right radial tunnel revealed a right PIN that was pinned down by the fascia and arcade of Frohse of the right supinator muscle. An incision was made along the dorsal border of the BR starting at the elbow crease and extending distally about 10 centimeters $(\mathrm{cm})$. The lateral cutaneous nerve of the forearm and the fascial plane between the BR and the ECRL was identified. This was opened and the dissection was carried out deeply. The radial nerve was identified proximally and no adhesions noted, even proximal to the elbow. Distally, the SRN and PIN were identified. There was significant compression of the 


\section{Cureus}

PIN by the arcade of Frohse at the proximal edge of the supinator. The compression was relieved by dissection and the PIN was tracked distally along the supinator to ensure no further bands of compression distal to the arcade (Figure 4).

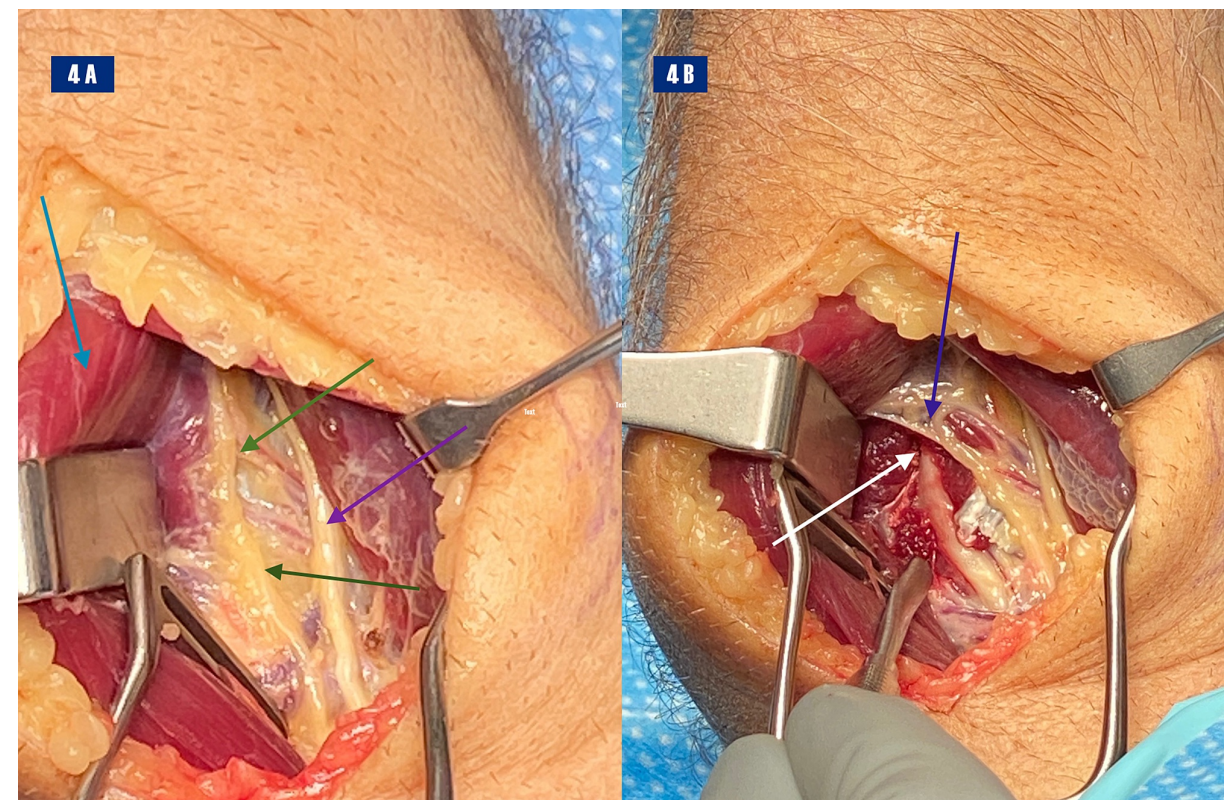

FIGURE 4: Right forearm incision: 4A: Deflection of right brachioradialis (light blue arrow), posterior interosseous nerve restrained by fibrous adhesions (green arrows), tendon of extensor carpi radialis brevis (purple arrow). 4B: Dissected arcade (blue arrow), posterior interosseous nerve (white arrow)

One month after surgery, the patient noted a definite but small improvement in finger extension. He was advised that recovery is a slow process and may take up to 12 months.

\section{Discussion}

A fascinating retrospective review of 15 patients by Maldonado et al., recommended exercising caution and a careful reanalysis of PIN syndrome in nontraumatic cases. Four patients had a well-defined mass compressing the PIN. The rest of the patients had MRI and clinical findings, such as atrophy and weakness, NCS/EMG findings, and nerve hypertrophy beyond the territory of the PIN. These findings reappraised the diagnosis to a more diffuse process such as an inflammatory neuropathy, as seen with a mononeuritis multiplex or a posterior cord of brachial plexus. They highlighted the manifold pathophysiologic classification of PIN lesions to compression, inflammation, and fascicular constriction [4].

Vrieling et al., in a study of 14 patients, distinguished between PIN paralysis and a radial tunnel syndrome, the latter presenting with the typical pattern of weakness plus forearm pain. They recommended an observation period of six to eight months in those patients with PIN paresis, prior to exploration [5].

Anania et al., in a review of seven cases, emphasized that entrapment distal to the arcade of Frohse is rare, reported in only one out of their seven cases [6]. Berton et al. studied cadavers and looked at the pennation pattern of the superficial and deep heads of the supinator. The proximal arcade was tendinous in almost two-thirds of cadavers and the distal arcade was tendinous in about three-quarters of cadavers. In between, there were no adhesions or tendinous lesions [7].

In a retrospective review by Cravens and Kline of over 15 years, PIN disorders represented 32 out of 170 radial nerve disorders. The table below classifies the lesions (Table 1) [8]. 


\section{Cureus}

\begin{tabular}{|l|l|}
\hline Lesion & Occurrence Number \\
\hline Entrapment at the arcade of Frohse & 14 \\
\hline Laceration & 6 \\
Fracture & 6 \\
Compression or contusion & 3 \\
\hline Tumor & 3 \\
\hline
\end{tabular}

TABLE 1: Table classifying PIN lesions in one of the largest retrospective studies.

PIN: Posterior interosseous nerve

Twenty-six patients underwent surgery including neurolysis of adhesions, decompression, excision of the local tumor, and in four cases of laceration; end-to-end suture repair, and rarely grafts. The overall prognosis was very good, with near recovery in most cases.

There are several case reports of proximal radio-ulnar ganglion lesions involving the PIN with excellent recovery following excision [9], of intraneural PIN lipomas [10], isolated case reports of PIN schwannomas [11], neuralgic amyotrophy presenting as an isolated PIN palsy [12], and rheumatoid arthritis with synovial invasion [13].

An interesting and not unusual differential diagnosis of finger drop is due to a cervical C8 nerve root lesion. The rationale being that the EDC has nerve supply from the cervical $\mathrm{C} 6, \mathrm{C} 7$, and $\mathrm{C} 8$ nerve roots. In a report of 17 cases, Koda et al. note that recovery was usually delayed by at least nine months after surgery, and surgical decompression was favored over conservative management [14].

It is conceivable that the PIN and radial nerve proper (supplying BR, ECRL, and triceps) are compartmentalized. A high-resolution ultrasound study of the radial nerve at the spiral groove revealed a figure of eight fascicular pairs that split distally in the upper arm [15]. Interestingly, a prior study of 26 patients with radial nerve, PIN and posterior cord brachial plexus injuries due to trauma and tumor, had identified dual pathology in the radial nerve proper and PIN, mostly focal nerve hypertrophy [16].

Furthermore, the profile of radial nerve injuries is different in the pediatric population. In a retrospective study of 19 children with traumatic nerve injuries of the radial nerve, the lesion was localizable to the PIN in $37 \%$ of cases, an unusually high frequency [17].

Bäumer et al. point out that PIN syndrome can be due to trauma, tumor, compression, or inflammation. They also report that partial lesions of the proximal radial nerve may mimic a PIN syndrome. They deployed magnetic resonance neurography to study 19 patients with PIN syndrome and observed T2-weighted hyperintense signal abnormalities of the proximal radial nerve in $84.2 \%$ of patients, with the larger proportion of radial nerves revealing a partial fascicular lesion. These lesions occurred in the deeper branch of the radial nerve that continued into the PIN. Some of these lesions also extended proximally into the posterior cord of the brachial plexus, and this may explain how an immune-mediated brachial plexopathy can cause a PIN syndrome. They also posit that a PIN syndrome can occur secondary to a more proximally placed lesion due to a selective fascicular lesion, with the PIN occupying a dorsomedial location along the radial nerve. They also speculate on a potential explanation for the intriguing phenotype of a "Texas longhorn" hand gesture, with selective middle and ring finger drop due to a length-dependent polyneuropathy, noting that the middle and ring fingers are the longest [18].

\section{Conclusions}

In summary, lesions of the PIN are quite variable and include tumors, trauma, inflammation, and compressive lesions. One can often obtain a "spot diagnosis" due to its unique clinical profile. Fascinating mimics include a C8 nerve root lesion or a high radial nerve lesion due to fascicular compartmentalization of the PIN and radial nerve proper. Its journey in the forearm is highlighted by useful landmarks including the radial neck, the proximal and distal arcades of the supinator muscle, where potential pathology may occur, as noted above.

\section{Additional Information}

Disclosures 
Human subjects: Consent was obtained or waived by all participants in this study. Conflicts of interest: In compliance with the ICMJE uniform disclosure form, all authors declare the following: Payment/services info: All authors have declared that no financial support was received from any organization for the submitted work. Financial relationships: All authors have declared that they have no financial relationships at present or within the previous three years with any organizations that might have an interest in the submitted work. Other relationships: All authors have declared that there are no other relationships or activities that could appear to have influenced the submitted work.

\section{References}

1. Spinner M: Injuries to the Major Branches of Peripheral Nerves of the Forearm . W.B. Saunders Company, Philadelphia; 1972.

2. Thomas SJ, Yakin DE, Parry BR, Lubahn JD: The anatomical relationship between the posterior interosseous nerve and the supinator muscle. J Hand Surg Am. 2000, 25:936-941. 10.1053/jhsu.2000.16360

3. Quignon R, Marteau E, Penaud A, Corcia P, Laulan J: Posterior interosseous nerve palsy. A series of 18 cases and literature review. Chir Main. 2012, 31:18-23. 10.1016/j.main.2011.11.004

4. Maldonado AA, Amrami KK, Mauermann ML, Spinner RJ: Nontraumatic "isolated" posterior interosseous nerve palsy: reinterpretation of electrodiagnostic studies and MRIs. J Plast Reconstr Aesthet Surg. 2017, 70:159-165. 10.1016/j.bjps.2016.11.017

5. Vrieling C, Robinson PH, Geertzen JHB: Posterior interosseous nerve syndrome: literature review and report of 14 cases. Eur J Plast Surg. 1998, 21:196-202. 10.1007/s002380050068

6. Anania P, Fiaschi P, Ceraudo M, Balestrino A, Zaottini F, Martinoli C, Gennaro S: Posterior interosseous nerve entrapments: review of the literature. Is the entrapment distal to the arcade of Frohse a really rare condition?. Acta Neurochir. 2018, 160:1857-1864. 10.1007/s00701-018-3615-8

7. Berton C, Wavreille G, Lecomte F, Miletic B, Kim H-J, Fontaine C: The supinator muscle: anatomical bases for deep branch of the radial nerve entrapment. Surg Radiol Anat. 2013, 35:217-224. 10.1007/s00276-0121024-x

8. Cravens G, Kline D: Posterior interosseous nerve palsies. Neurosurgery. 1990, 27:397-402. 10.1097/00006123-199009000-00009

9. McCollam SM, Corley FG, Green DP: Posterior interosseous nerve palsy caused by ganglions of the proximal radioulnar joint. J Hand Surg Am. 1988, 13:725-728. 10.1016/S0363-5023(88)80134-5

10. Patel AP, Aoun SG, Tamimi MA: Intraneural posterior interosseous nerve lipoma with complete paralysis: case report and review of the literature. Cureus. 2018, 10:e2689. 10.7759/cureus.2689

11. Ichikawa J, Sato E, Haro H, Anayama S, Ando T, Hamada Y: Posterior interosseous nerve palsy due to schwannoma: case report. J Hand Surg Am. 2008, 33:1525-1528. 10.1016/j.jhsa.2008.05.033

12. Yang JS, Cho YJ, Kang SH, Choi EH: Neuralgic amyotrophy manifesting as mimicking posterior interosseous nerve palsy. J Korean Neurosurg Soc. 2015, 58:491-493. 10.3340/jkns.2015.58.5.491

13. Chan JK, Kennett R, Smith G: Posterior interosseous nerve palsy in rheumatoid arthritis: case report and literature review. J Plast Reconstr Aesthet Surg. 2009, 62:E556-E560. 10.1016/j.bjps.2008.10.013

14. Koda M, Furuya T, Rokkaku T, et al.: Drop finger caused by 8th cervical nerve root impairment: a clinical case series. Eur Spine J. 2017, 26:1096-1100. 10.1007/s00586-016-4836-2

15. Gamber D, Motte J, Kerasnoudis A, Yoon M-S, Gold R, Pitarokoili K, Fisse AL: High-resolution nerve ultrasound to assess nerve echogenicity, fascicular count, and cross-sectional area using semiautomated analysis. J Neuroimaging. 2020, 30:493-502. 10.1111/jon.12717

16. Dietz AR, Bucelli RC, Pestronk A, Zaidman CM: Nerve ultrasound identifies abnormalities in the posterior interosseous nerve in patients with proximal radial neuropathies. Muscle Nerve. 2016, 53:379-383. 10.1002/mus.24778

17. Karakis I, Georghiou S, Jones HR, Darras BT, Kang PB: Electrophysiologic features of radial neuropathy in childhood and adolescence. Pediatr Neurol. 2018, 81:14-18. 10.1016/j.pediatrneurol.2018.01.003

18. Bäumer P, Kele H, Xia A, Weiler M, Schwarz D, Bendszus M, Pham M: Posterior interosseous neuropathy: supinator syndrome vs fascicular radial neuropathy. Neurology. 2016, 87:1884-1891. 10.1212/WNL.0000000000003287 